This item was submitted to Loughborough's Research Repository by the author.

Items in Figshare are protected by copyright, with all rights reserved, unless otherwise indicated.

\title{
The design and characterization of multifunctional aptamer nanopore sensors
}

PLEASE CITE THE PUBLISHED VERSION

https://doi.org/10.1021/acsnano.8b01583

PUBLISHER

(C) American Chemical Society

VERSION

AM (Accepted Manuscript)

\section{PUBLISHER STATEMENT}

This work is made available according to the conditions of the Creative Commons Attribution-NonCommercialNoDerivatives 4.0 International (CC BY-NC-ND 4.0) licence. Full details of this licence are available at: https://creativecommons.org/licenses/by-nc-nd/4.0/

\section{LICENCE}

CC BY-NC-ND 4.0

\section{REPOSITORY RECORD}

Mayne, Laura J., Chih-Yuan Lin, Steven Christie, Zuzanna S. Siwy, and Mark Platt. 2018. "The Design and Characterization of Multifunctional Aptamer Nanopore Sensors". Loughborough University.

https://hdl.handle.net/2134/33172. 
The Design and Characterisation of Multifunctional Aptamer Nanopore Sensors

Laura Mayne ${ }^{\mathrm{a}, \mathrm{e}}$, Chih-Yuan Lin ${ }^{\mathrm{b}, \mathrm{e}}$, Steven D. R. Christie ${ }^{\mathrm{a}}$, Zuzana S. Siwy ${ }^{\mathrm{b}, \mathrm{c}, \mathrm{d \#}}$, Mark Platt $^{\mathrm{a}^{*}}$

a. Department of Chemistry, Loughborough University, Loughborough, UK.

b. Department of Physics and Astronomy, University of California, Irvine, USA

c. Department of Chemistry, University of California, Irvine, USA

d. Department of Biomedical Engineering, University of California, Irvine, USA

e. We wish to note that these authors contributed equally to the work in the paper

\$s.d.christie@lboro.ac.uk

\# zsiwy@uci.edu

* corresponding Author m.platt@lboro.ac.uk 


\begin{abstract}
Aptamer modified nanomaterials provide a simple, yet powerful sensing platform when combined with Resistive Pulse Sensors, RPS, technologies. Aptamers adopt a more stable tertiary structure in the presence of a target analyte which results in a change in charge density and velocity of the carrier particle. In practice the tertiary structure is specific for each aptamer-target, and the strength of the signal varies with different applications and experimental conditions. RPS have single particle resolution, allowing for the detailed characterisation of the sample. Measuring the velocity of aptamer-modified nanomaterials as they traverse the RPS provides information on their charge state and densities. To help understand how the aptamer structure and charge density effects the sensitivity of Aptamer-RPS assays, here we study two metal binding aptamers. This creates a sensor for mercury and lead ions that is capable of being run in a range of electrolyte concentrations, equivalent to river to sea water conditions. The observed results are in excellent agreement with our proposed model. Building on this we combine two aptamers together in an attempt to form a dual sensing strand of DNA for the simultaneous detection of two metal ions. We show experimental and theoretical responses for the aptamer which creates layers of differing charge densities around the nanomaterial. The density and diameter of these zones effects both the viability and sensitivity of the assay. Whilst this approach allows the interrogation of the DNA structure, the data also highlights the limitations and considerations for future assays
\end{abstract}

keywords: resistive pulse sensor, aptamer, sensor, electrophoretic mobility, DNA structure. 
Aptamers are sequence specific single strand nucleic acids, that bind to target analytes via the formation of a specific tertiary structure. ${ }^{1,2}$ They are typically isolated from chemically synthesised oligonucleotide libraries containing $10^{4}-10^{15}$ different sequences. $^{3-5}$ The process was first reported in the early 1990's and since the discovery of the in vitro selection process, they have been increasingly advocated as alternatives to antibodies. ${ }^{6}$ They have applications as therapeutics, bioimaging and as recognition elements in many sensing platforms. ${ }^{7,8}$

The chemical versatility of DNA allows for modified bases to be incorporated into their secondary structure, endowing them with beneficial properties. Many sensing applications utilise this in combination with the change in tertiary structure to create sensors and comprehensive reviews on this can be found elsewhere..$^{8-11}$ Insertion of probes or modified bases into the secondary structure must be done with some prior knowledge of the final tertiary structure, or there is a risk of changing the specificity, affinity or at worst destroying the recognition of the aptamer altogether. Thus there has been a move towards "tagless" systems where the binding of the analyte can be monitored without the use of probes. A range of sensing platforms have emerged, often they choose similar targets to validate the technologies. This is due to the availability of the Aptamers themselves or the preference to choose known systems that have high affinities. Given that each target and aptamer pair adopt a specific structure, technologies that rely upon large changes to tertiary structure may not offer a universal sensing platform for future analytes.

One emerging technology that is well suited for studying DNA in its natural form, is Resistive Pulse Sensing, RPS. It allows the characterisation of DNA sequence, structure and chemical changes as they translocate the nanopore. ${ }^{12,13}$ The incorporation of Aptamer sequences into this technology has further expanded the repertoire of RPS. ${ }^{14-16}$ The translocation of analytes through these channels, or nanopores can be monitored by measuring the ionic current. Each translocation event causes a change to the conductivity of the channel, which is related to the physical properties of the analyte and information on the size, concentration and charge can be measured quickly. ${ }^{17-20}$ Aptamer based RPS strategies can be broadly divided into three areas. The first is the modification of the pore mouth/ walls with aptamers to induce a change in current flow upon the binding. Second, measuring the translocation rates and velocities of the aptamer sequences through the pores, or finally the use of nanomaterials as carriers for multiple aptamers. ${ }^{15,21-24}$

The latter is appealing when the nanomaterials are Superparamagnetic Beads, SPB's, as the particles can perform both the purification and sensing roles. By adopting a tertiary structure in the presence of the target, the charge density around the SPB is altered. ${ }^{24,25}$ This is measured through a change in translocation velocity and can provide quantitative information. ${ }^{26-28}$ However RPS signals can be affected by the experimental setup. Studies have shown that ionic strength, off-axial translocation, the pore wall charge density and applied potential can affect the pulse size and width. ${ }^{29-32}$ In cases where the charge double layers around the particles and pore walls interact, conductive pulses and ion current rectification can also be observed. ${ }^{21,33,34}$

To help understand how the charge density effects the sensitivity of Aptamer RPS assays here we choose two known aptamers to heavy metal ions. The field of DNA-Metal ion sensors is growing and comprehensive review of the topic can be found elsewhere. ${ }^{35-37}$ Here two common examples are chosen namely Lead $\left(\mathrm{Pb}^{2+}\right)$ and Mercury $\left(\mathrm{Hg}^{2+}\right)$. These represent two examples that have biological and environmental importance, as well as two different and known tertiary structures. 
Oligonucleotides which are $\mathrm{G}$ rich are known to form G-quadruplex structures in the presence of specific metal ions. ${ }^{38,39}$ The strong association of $\mathrm{Pb}^{2+}$ within the Gquadruplex's has made it possible to develop sensors, and is the first aptamer. ${ }^{37}$ The second chosen aptamer is a $\mathrm{T}$ rich single stranded DNA which binds $\mathrm{Hg}^{2+}$. Upon the binding of $\mathrm{Hg}^{2+}$ to the sequence it folds in to a hairpin due to the $\mathrm{T}-\mathrm{Hg}^{2+}-\mathrm{T}$ coordination.

First we illustrate a simple assay using Aptamer modified SPBs and RPS to detect $\mathrm{Pb}^{2+}$ and $\mathrm{Hg}^{2+}$ with $\mathrm{nM}$ sensitivity. The methodology works across a wide range of electrolyte concentrations. The experimental data fits the predicted model and calculations which show how the distribution of metal ions and charge density around the SPBs determine the signal strength. Our measurements and modelling show that the charge density of G-quadruplex is lower compared to the charge of unfolded DNA; the lowering of the charge density is attributed to the specific binding of stabilizing cations, including $\mathrm{K}^{+}$and divalent ions. ${ }^{40,41}$ We also present simulations for signal strength when the aptamer length and relative location of the tertiary structure and flanking nonbinding SSDNA to the surface of the SPB are changed. This insight gives a basis to understand how to design and predict the behaviour of future RPS aptamer assays. The assay can also be multiplexed via the use of different sized SPBs, each one equipped with a different aptamer, and we show a facile multiplexed assay for the simultaneous detection of two metal ions. In an attempt to advance this multiplexed concept we merge two independent aptamer sequences together. Whilst both aptamer halves retain their initial functionality and bind to the respective metals, the location of the binding and change in DNA structure with respect to the particles surface is the dominating factor in determining the sensitivity of the RPS technology. This observation is backed with modelling and offers a description and understanding and predicating the behaviour of future RPS assays.

\section{Results and Discussion}

To relate the structure and length of the aptamer, and its effect on the relative velocity through the RPS, we opted to use aptamers that bind to metal ions. This allows us to remove any effects from the added complications of the size and charge of protein/ cell targets, as well as its isoelectric point in the buffer used. The interaction between the two aptamers and their respective targets of $\mathrm{Hg}^{2+}$ and $\mathrm{Pb}^{2+}$ have been well studied. The $\mathrm{Pb}$ aptamer forms a G-quadruplex where each aptamer holds a single ion within the quadruplex cage, ${ }^{42}$ figure $1 \mathrm{a}$. The $\mathrm{Hg}$ aptamer forms into a stem-loop structure holding up to seven metal ions within the stem region bound between two thymine residues, ${ }^{43}$ figure $1 \mathrm{a}$.

In the first experiments two identical sets of $150 \mathrm{~nm}$ SPB beads were each functionalised with a metal binding aptamer. This produced one batch of beads functionalised with the $\mathrm{Pb}$ aptamer and a second batch functionalised with the $\mathrm{Hg}$ aptamer. The presence of the aptamer was inferred by following the relative velocity of the particles through the functionalisation stages as described elsewhere. ${ }^{44}$ First the $\mathrm{Pb}$ aptamer beads were incubated with a range of $\mathrm{Pb}^{2+}$ concentrations. As shown in figure $1 \mathrm{c}$ as the $\mathrm{Pb}^{2+}$ concentration increases the relative velocity of the particle decreases, examples of raw data are given on figure S1. We attribute this velocity change to the change in charge density around the particles, which was probed before via the dependence of G-quadruplex's melting temperature, $T_{M}$, on cation concentration. ${ }^{40,41}$ The decreased charge density of G-quadruplex compared 
to unfolded DNA is due to both (i) specific ion binding, in our case $\mathrm{Pb}$ as well as potentially $\mathrm{Na}^{+}$ions, which also were found to stabilize the G-quadruplex, and (ii) ion condensation. It is important to note that only one $\mathrm{Pb}^{2+}$ ion binds per aptamer, and the velocity decrease can be observed even for $1 \mathrm{nM}$ of $\mathrm{Pb}^{2+}$ added; the reduction in particle velocity with the increase of the metal ion concentration can be seen across a wide range of ionic strengths. To check this effect was not specific to the $\mathrm{NaCl}$ buffer, similar experiments were run in $\mathrm{KCl}$ shown in figure $\mathrm{S} 2 \mathrm{a}$. The $\mathrm{Hg}$ aptamer functionalised beads were then tested, and the data is shown in figure 1d. Again as the $\mathrm{Hg}^{2+}$ concentration increases the velocity of the particles decreases. Control experiments showing the relative velocity of the aptamers in the presence of a nonbinding metal ion are shown in figure S1b and c, illustrating the effect is specific to the heavy metal ions. Lack of sensitivity of the aptamer modified beads to a nonbinding ion (particles with $\mathrm{Pb}$ binding aptamer were exposed to $\mathrm{Hg}$, and particles with $\mathrm{Hg}$ binding aptamer were exposed to $\mathrm{Pb}$ ) suggests that binding of the divalents affects charge of the particles only, i.e. $\mathrm{Pb}^{2+}$ or $\mathrm{Hg}^{2+}$ does not participate in the process of screening surface charges of the pore wall. If the pore surface charges were more screened after addition of the divalent ions, the reduced electroosmotic velocity would also lead to slowing down of the particles, and would be observed in Figure S2b,c.

In both cases, of $\mathrm{Pb}$ and $\mathrm{Hg}$ binding aptamers, the particles were added at similar concentrations, and thus it's interesting to note the range of metal ion concentrations that cause a response for each particle set. For the $\mathrm{Pb}$ aptamer, concentrations less than $10 \mathrm{nM}$ cause a decrease in particle velocity, and the signal seems to remain steady between $10-20 \mathrm{nM}$. This may indicate all the metal binding site are occupied or the technique can no longer resolve the change in velocity. For the $\mathrm{Hg}$ aptamer a much higher concentration of $\mathrm{Hg}^{2+}$ is needed before a decrease in velocity is recorded circa $10-20 \mathrm{nM}$, and the particle velocity continues to decrease up to $200 \mathrm{nM}$. As the concentration of the particles, and the density of DNA on their surfaces is similar, the difference in the $\mathrm{Hg}^{2+}$ binding curves are attributed to the ability of the $\mathrm{Hg}$ aptamer DNA to bind to multiple metal ions simultaneously. This may require higher levels of $\mathrm{Hg}^{2+}$ to be present before a stable Stem-loop is formed, and because each DNA can bind up to seven $\mathrm{Hg}^{2+}$ ions the dynamic range is extended by circa 7 times that of $\mathrm{Pb}^{2+}$. These results suggest that binding of the ions, even only one $\mathrm{Pb}^{2+}$ ion per aptamer, leads to detectable change of the aptamer charge.

a
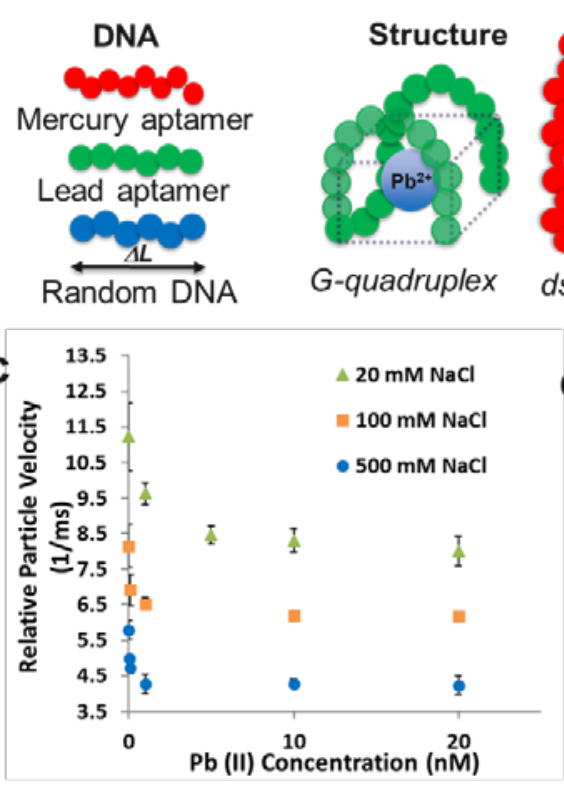

b

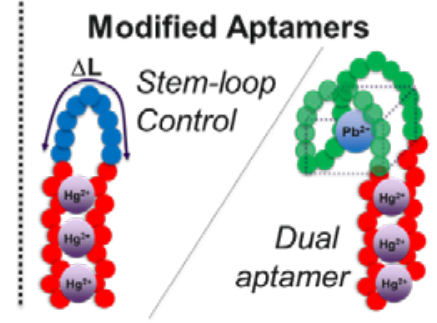

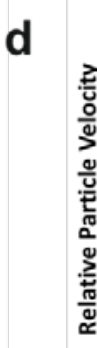




Figure 1. a) Schematic of the DNA used in the study and the expected structure. b) Dual DNA structures where the length of the loop (DL) and two aptamers are combined. Plots of relative particle velocity versus ion concentration for c) $\mathrm{Pb}^{2+}$ and d) $\mathrm{Hg}^{2+}$. SPB were $150 \mathrm{~nm}$ in diameter.
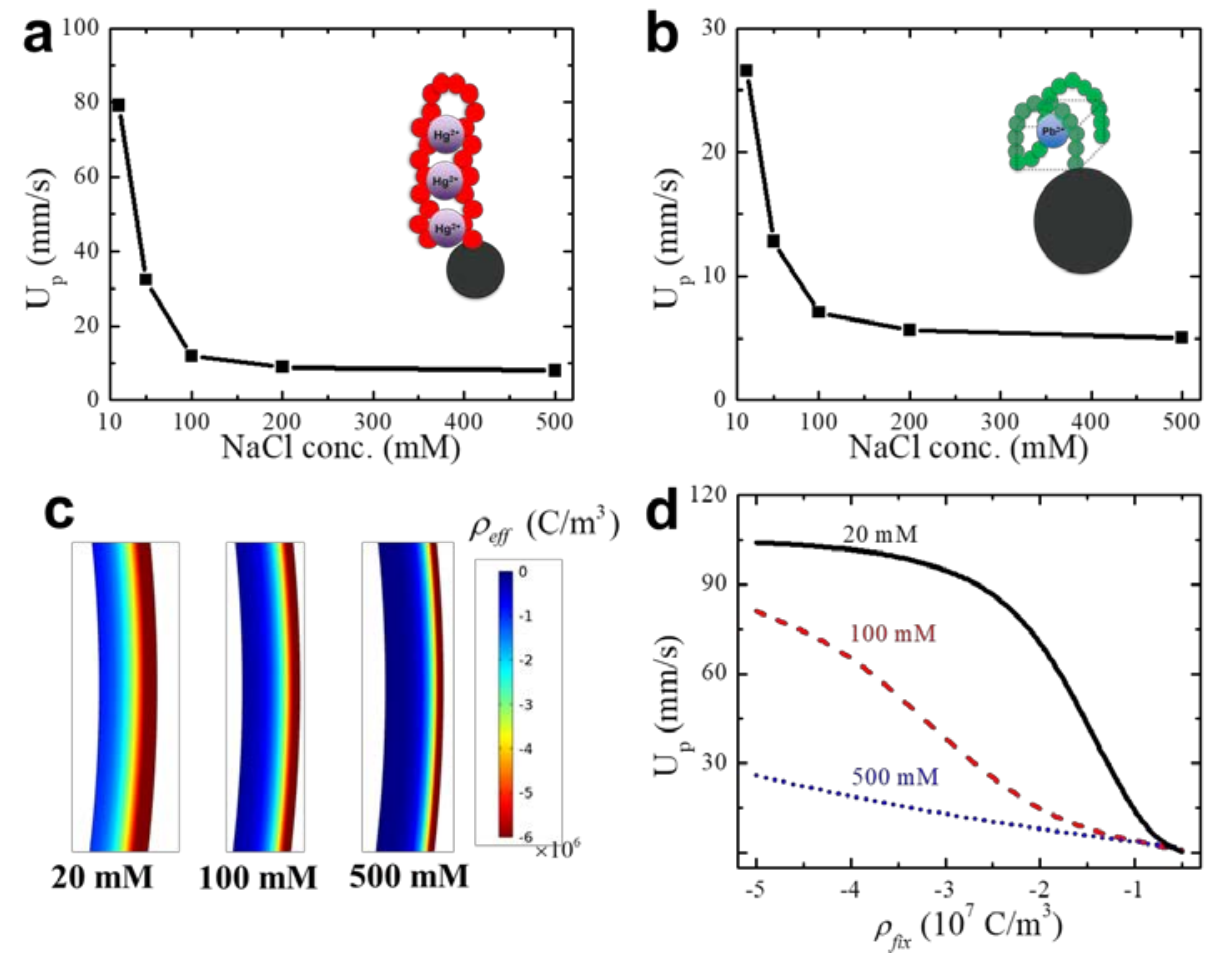

Figure 2. Variation of the translocation velocity, $U_{p}$, of DNA modified particle as a function of the bulk $\mathrm{NaCl}$ concentration for different sized particles with charge density of $-2 \times 10^{7} \mathrm{C} / \mathrm{m}^{3}$. a) $150 \mathrm{~nm}$ with $3 \mathrm{~nm}$ soft layer; b) $300 \mathrm{~nm}$ with $2 \mathrm{~nm}$ soft layer. c) Spatial distribution of the effective charge density $\rho_{\text {eff }}$ at various bulk $\mathrm{NaCl}$ concentrations for the case of Figure $2 \mathrm{a} . \mathrm{d}$ ) Dependence of the translocation velocity of DNA modified particles as a function of the charge density $\rho_{\text {fix }}$ at varying concentration of $\mathrm{NaCl}$. The pore geometry and thickness of the DNA layer were chosen to match the experimental parameters. The tip diameter of the pore was $700 \mathrm{~nm}$, the particle diameter was $150 \mathrm{~nm}$, and the thickness of the soft DNA layer was assumed $3 \mathrm{~nm}$. The modelling was done assuming electric potential difference of $0.6 \mathrm{~V}$.

To understand this effect, we modelled the translocation velocity, $U_{p}$, of a particle across a range of salt concentrations. Figure 2 shows the velocity data for a) $150 \mathrm{~nm}$ 
and b) $300 \mathrm{~nm}$ in diameter particles, where the $\mathrm{NaCl}$ concentration ranged between $20 \mathrm{mM}$ and $500 \mathrm{mM}$. Higher salt concentrations induced accumulation of ions within a smaller distance from the surface (shorter Debye length, figure 2c) compared to more diluted solutions, leading to effectively lower charge density thus lower electrophoretic velocity. Next we simulate the binding of the metal ions to the DNA. Figure $2 \mathrm{~d}$ shows the velocity versus particle charge density from $-5 \times 10^{7} \mathrm{C} / \mathrm{m}^{3}{ }^{45-48}$ corresponding to an estimate for no divalent ions bound to DNA, and $-0.5 \times 10^{7} \mathrm{C} / \mathrm{m}^{3}$. We considered the rather large range of charge densities to demonstrate that even a small change of charge density is predicted to lead to detectable change of the particle velocity, calculation can be found in the supplementary section. The modelling is therefore in agreement with the experimental findings, since the velocity decreased when only one $\mathrm{Pb}^{2+}$ ion bound to the $\mathrm{Pb}$ specific aptamer as well as when $7 \mathrm{Hg}^{2+}$ ions bound to the $\mathrm{Hg}$ specific aptamer. The modelling also suggested that the translocation velocity is more sensitive to changes in charge density of the particles at low salt concentrations. The effect of salt concentration on translocation is also in agreement with lower zeta potential of the particles at high salt concentrations.

a

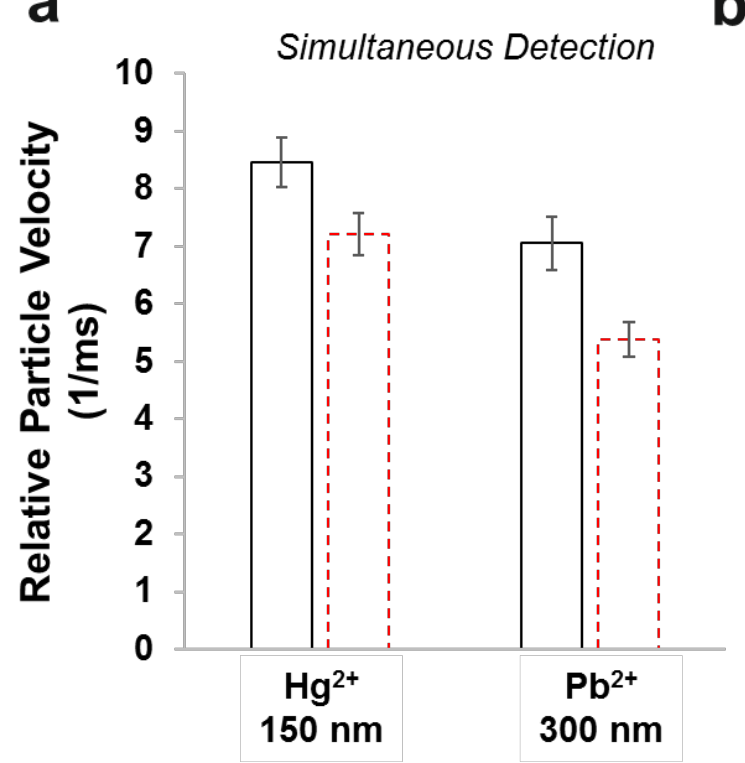

b

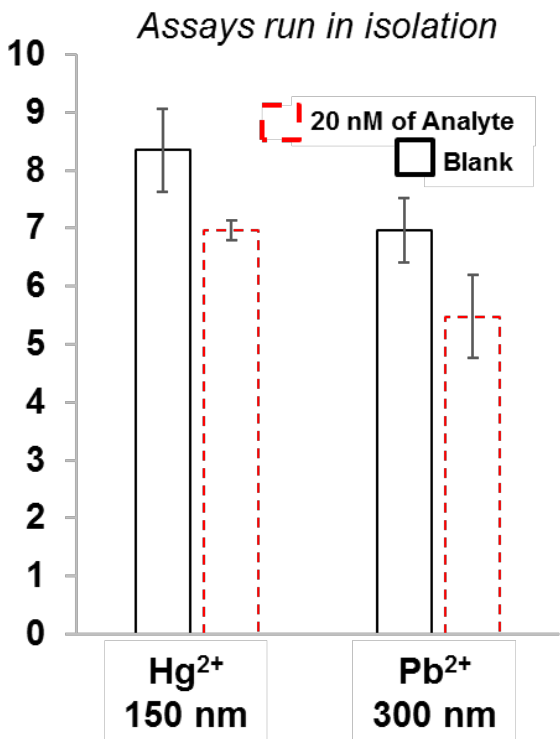

Figure 3. $\mathrm{Hg}^{2+}$ and $\mathrm{Pb}^{2+}$ binding aptamers placed onto 150 and $300 \mathrm{~nm}$ particles respectively. Both particles were present in solution and $20 \mathrm{nM}$ metal ions added a) simultaneously, and b) individually. The data in (b) represents two assays. In the first assay, $150 \mathrm{~nm}$ particles modified with $\mathrm{Hg}^{2+}$ specific aptamer were subjected to $\mathrm{Hg}^{2+}$ only; in the second assay, $400 \mathrm{~nm}$ in diameter particles with $\mathrm{Pb}^{2+}$ specific aptamer were subjected to a solution containing $\mathrm{Pb}^{2+}$. All experiments were performed in $20 \mathrm{mM} \mathrm{NaCl}$ as the background electrolyte.

The data within figure 1 illustrated the ability of the RPS and aptamer functionalised particles to detect the presence of specific metal ions across a range of ionic strengths. One particularly powerful property of RPS sensors is their ability to distinguish particles based upon their size. This has allowed them to characterise multiple sized particles in the same solution, ${ }^{49}$ and has produced multiplexed bioassays. ${ }^{23}$ To illustrate the same effect here we functionalise two different sized 
particles, each with a specific aptamer. Here we place the $\mathrm{Hg}$ aptamer onto a particle (150 nm in diameter), and the $\mathrm{Pb}$ aptamer onto a larger particle (300 nm in diameter). Figure $3 a$ illustrates the change in velocity when both particle sets are present in same solution, from the blank (black box) to the addition of $20 \mathrm{nM}$ metal ions (red dashed box). For comparison, figure $3 b$ shows the same experiment when each assay is run independently i.e. Pb assay in one pot, and the $\mathrm{Hg}$ assay in a second. The results are comparable illustrating that neither the other particle or metal ion effects the assay.

The ability to quickly detect and quantify metal ions in solution is attractive, and has many applications within environmental and health sciences. But a typical RPS device will have an upper limit to the number of particles which can be run simultaneously. This is dependent upon the pore size, and ability to produce uniform beads within the pores sensing range. One way to increase the number of analytes for detection may be to create dual responsive beads i.e. one bead whose velocity changes in the presence of two different analytes. Two approaches can be envisaged for this. The first would be to mix two different aptamers together and place them both onto the beads surface. However a drawback to this approach is that both aptamers would need to be of similar length and reactivity to ensure an equal distribution on the beads surface, and extensive work has been done to illustrate the effects of ligand length and the binding kinetics onto surfaces. ${ }^{50}$ Here we propose and test an alternative, by combining the two aptamers above into one single dual responsive aptamer. G-quadruplex structures have been added to the end of stem regions previously which have been shown to enhance their binding to proteins. ${ }^{51}$ As shown in figure $1 \mathrm{~b}$ here we combine the two metal aptamers into one structure.

We placed this dual aptamer onto the $150 \mathrm{~nm}$ particles and incubated them with $\mathrm{Pb}^{2+}$ and/ or $\mathrm{Hg}^{2+}$. As can be seen in figure $4 \mathrm{a}$, the velocity of the particles only changed in the presence of $\mathrm{Pb}^{2+}$. No significant change in velocity was recorded for the dual aptamer even when $\mathrm{Hg}^{2+}$ was the only ion present, figure $4 \mathrm{a}$. To ascertain if the $\mathrm{Hg}$ section of the aptamer was still functional we performed a control experiment. The dual aptamer beads were first incubated in a solution containing $200 \mathrm{nM} \mathrm{Hg}{ }^{2+}$. After an hour, the dual aptamer particles were removed, and to the same solution fresh beads modified only with the $\mathrm{Hg}$ specific aptamer were added. The $\mathrm{Hg}$ aptamer modified beads were then subjected to RPS experiment, i.e. we measured the relative velocity of the beads, shown in figure $4 \mathrm{a}$. We observed only a small change in velocity, $8 \%$ compared to $36 \%$, recorded for the single aptamer beads shown figure 1 . We attribute this reduction in signal to be due to the initial dual aptamer particles binding the majority of the $\mathrm{Hg}^{2+}$ ions from the solution. Thus the second batch of particles, containing only the $\mathrm{Hg}$ binding aptamer, were subjected to a reduced $\mathrm{Hg}^{2+}$ concentration, leading to a reduction of the particles velocity.

In order to corroborate the claim of $\mathrm{Hg}^{2+}$ being bound by the dual aptamer, we considered the $\mathrm{Hg}^{2+}$ ions and particles' concentrations as well as DNA packing density. At a concentration of $\mathrm{Hg}^{2+}$ of $200 \mathrm{nM}$, and particles at $1 \times 10^{9} / \mathrm{mL}$ there are circa $1 \times 10^{5}$ ions per particle in solution. Using a moderate packing density of DNA on the carboxyl surface of $1 / 10 \mathrm{~nm}^{52}$ gives circa 700 ssDNA per particle and a ratio of 170 ions per DNA. Whilst this is in excess as each aptamer binds up to 7 ions per strand, the reduction in $\mathrm{Hg}^{2+}$ ions concentration from the first hybridisation will reduce the number of $\mathrm{Hg}^{2+}$ bound as dictated by the aptamer binding constant of $10^{6}$ $\mathrm{M}^{-1} \cdot 43$ 
Our experiments with particles modified with a dual aptamer suggested that the particles are sensitive only to ions that bind to the outer aptamer. This observation is both interesting and potentially harmful for RPS sensors, as it suggests that changes to the aptamer structure close to the beads surfaces maybe have little effect on the relative particle velocity.
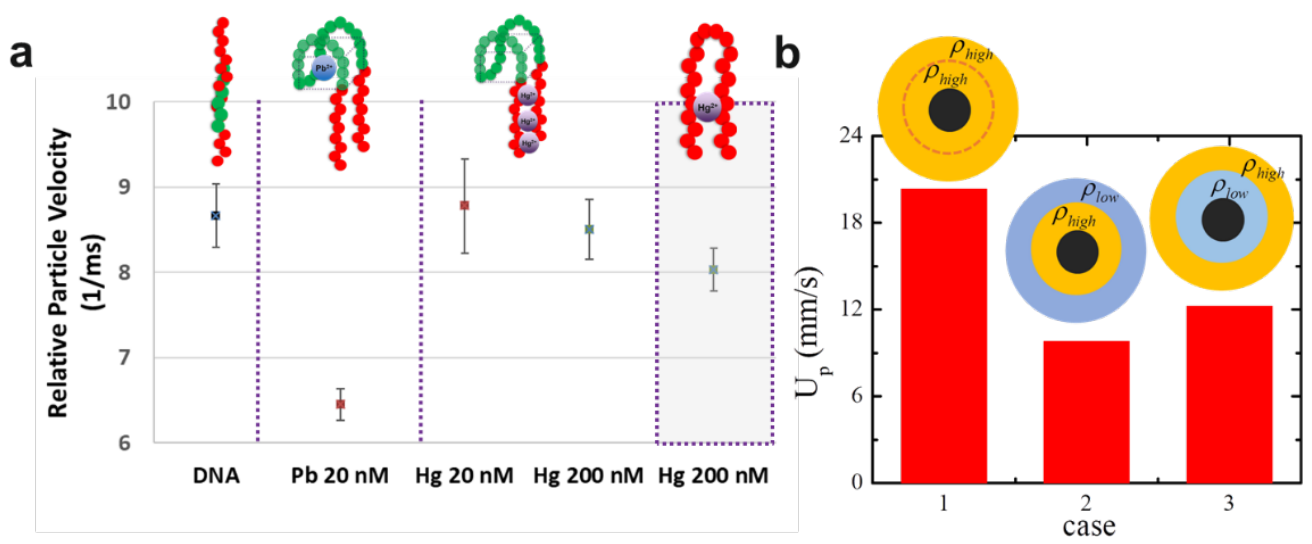

Figure 4. a) Measure of relative particle velocities for $150 \mathrm{~nm}$ particles functionalised with the dual aptamer, or single aptamer (shaded grey) incubated with target ions. b) Simulated translocation velocity containing two layers with thicknesses of $3 \mathrm{~nm}$ (the inner layer) and 2 $\mathrm{nm}$ (the outer layer). The $3 \mathrm{~nm}$ thick layer corresponds to the aptamer binding $\mathrm{Hg}^{2+}$, the $2 \mathrm{~nm}$ thick layer corresponds to the $\mathrm{Pb}^{2+}$ layer. The velocity was calculated for three cases: (1) both layers have equal charge of $-2 \times 10^{7} \mathrm{C} / \mathrm{m}^{3}$, (2) the charge density of the outer layer was decreased to $-1 \times 10^{7} \mathrm{C} / \mathrm{m}^{3}$, (3) the charge density of the inner layer was decreased to $-1 \times 10^{7}$ $\mathrm{C} / \mathrm{m}^{3}$.

To understand the implication of this observation we modelled the electrokinetic behaviour of particles modified with a combined double aptamer. These particles contained two layers one that could bind both mercury and lead. The inner layer had a thickness of $3 \mathrm{~nm}$ and corresponded to the mercury binding part of the double aptamer. The other layer was $2 \mathrm{~nm}$ thick and represented the aptamer that was specific to bind lead. Translocations of such particles were considered in three cases. In case 1 , both layers had the same charge density of $-2 \times 10^{7} \mathrm{C} / \mathrm{m}^{3}$. In case 2 , the charge density of the outer layer was decreased to $-1 \times 10^{7} \mathrm{C} / \mathrm{m}^{3}$. Finally, in case 3 , the density of the outer layer was kept at $-2 \times 10^{7} \mathrm{C} / \mathrm{m}^{3}$, but the density of the inner layer was lowered to $-1 \times 10^{7} \mathrm{C} / \mathrm{m}^{3}$, figure $4 \mathrm{~b}$. Case 2 therefore was our model system for the experimental situation in which lead bound to the double aptamer, while case 3 corresponded to the situation when only mercury was bound. The modelling qualitatively reproduced the experimental observations: reducing the charge of the outer layer had a significantly larger effect on the spheres translocation compared to the modulations of the charge of the inner layer.

In order to understand the role of the two layers in the electrokinetic transport of the particles, we looked in detail at the distribution of local ionic concentrations in both layers and outside the particle (figure 5). Note that at the interfaces of soft layer and soft layer/liquid, the concentration of each ionic species is continuous. ${ }^{53,54}$ The modelling revealed that ionic concentrations outside the particle are influenced by 
the charge density of the outer layer only; ionic concentrations are the same in case 1 and case 3 (see radial positions $>80 \mathrm{~nm}$ ). The electrokinetic velocity however is dependent on the ionic concentrations within the whole thickness of the outer layer. Average ionic concentrations across the entire outer layer in case 3 is lower than in case 1, and indeed the particle velocity is lower in case 3 . In case 2, ionic concentrations in the outer later are significantly lower than in cases $1 \& 3$ so that translocation velocity was the lowest. The secondary role of the inner layer for particle mobility is especially evident when looking at ionic concentrations in case 2 . The inner layer features ionic concentrations nearly as high as in case 1 , but it is the low ionic concentration in the outer layer, which is most probably responsible for the low mobility.
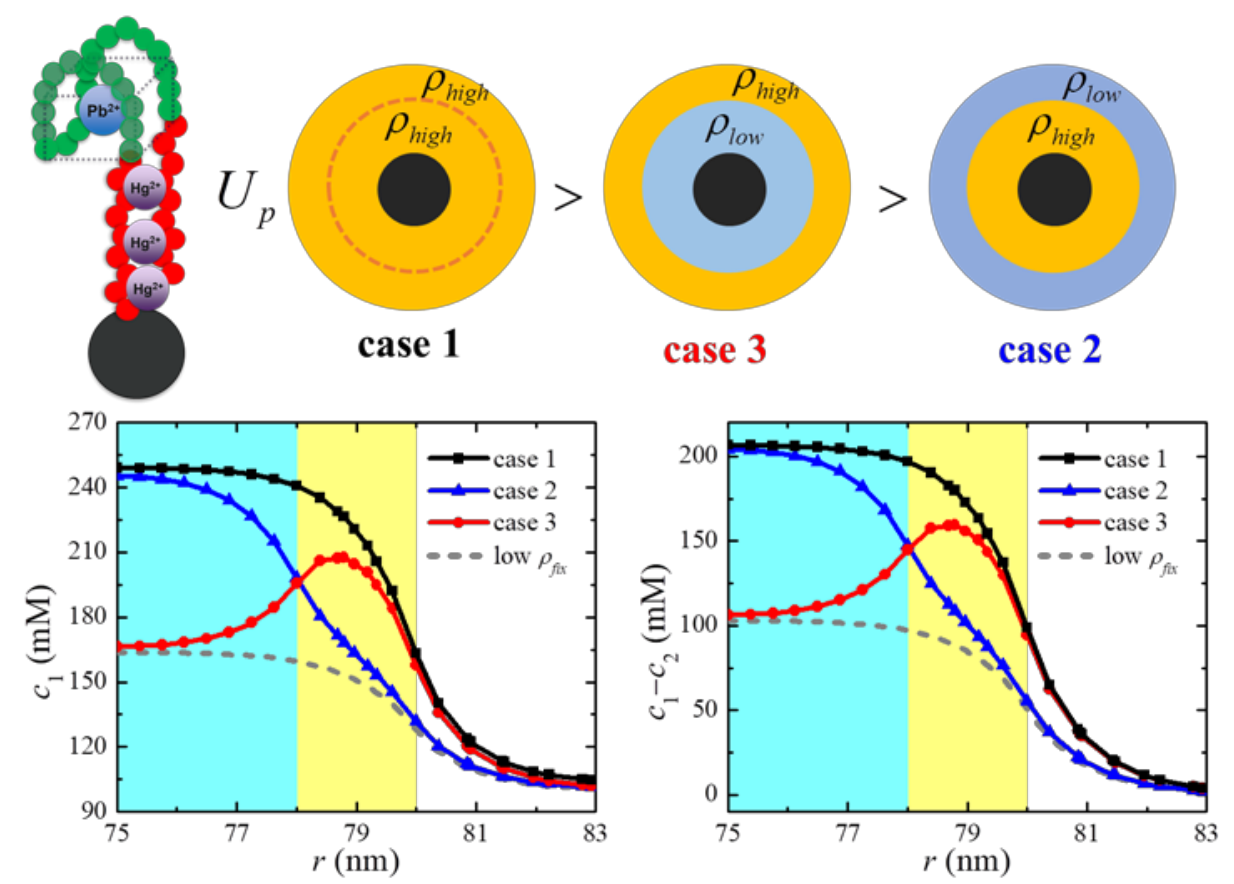

Figure 5. Variation of the cationic concentration, $c_{1}$, and concentration difference, $c_{1}-c_{2}$, along the $r$ direction of the soft layer for different cases. The blue and yellow regions denote the inner and outer layer of the dual aptamer modified nanoparticle, respectively.

We have also calculated volume-averaged charge densities for case 2 and case 3 taking into account both layers. Table S1 shows average charge densities calculated as the integrated charge in the inner and outer layers divided by their summed volumes. The average charge density for case 2 is higher than in case 3 , even though the translocation velocity exhibits the opposite effect. Thus, these calculations also underline the determinant role of the outer layer in the electrokinetic passage.

To further test this theory, we designed a control experiment where the loop length of the initial $\mathrm{Hg}^{2+}$ aptamer was varied from 4 (the original length) to 15 bases (the Gquadruplex), figure $1 \mathrm{~b}$, and the data is presented in figure $6 \mathrm{a}$. The data in figure 6 shows the relative velocities for the DNA (Aptamer with no metal ion present) and when $200 \mathrm{nM} \mathrm{Hg}^{2+}$ is added to the solution. A loop length of 4 represents the original aptamer sequence, increasing the loop length results in a diminishing response and change between the blank and assay signal. i.e. as the outer layer of 
DNA become longer the change in signal decreases. This observation may indicate there is a limit to what aptamer structure and length can be observed in RPS sensors. Finally, we modelled the experimental case in which the thickness of the outer layer was systematically varied from $1 \mathrm{~nm}$ to $6 \mathrm{~nm}$. We wanted to probe whether the sensitivity of the dual aptamer to mercury diminishes when the outer layer becomes thicker. The experimental trend was reproduced in the modelling semi-quantitatively. Indeed, with the increase of the outer layer width, the relative change of the particle velocity before and after adding mercury diminishes.

a

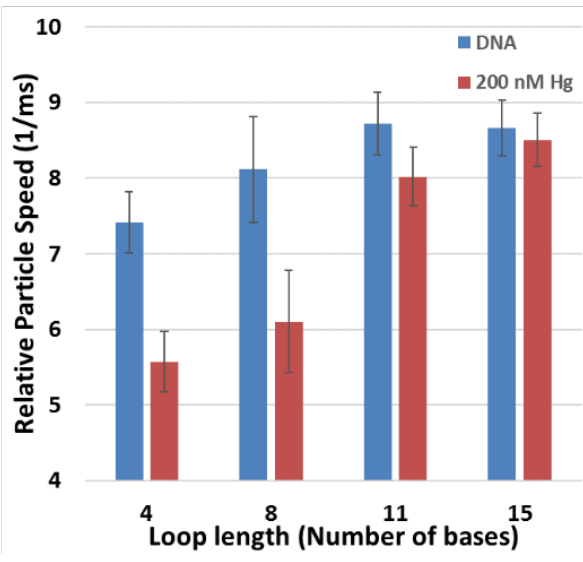

b

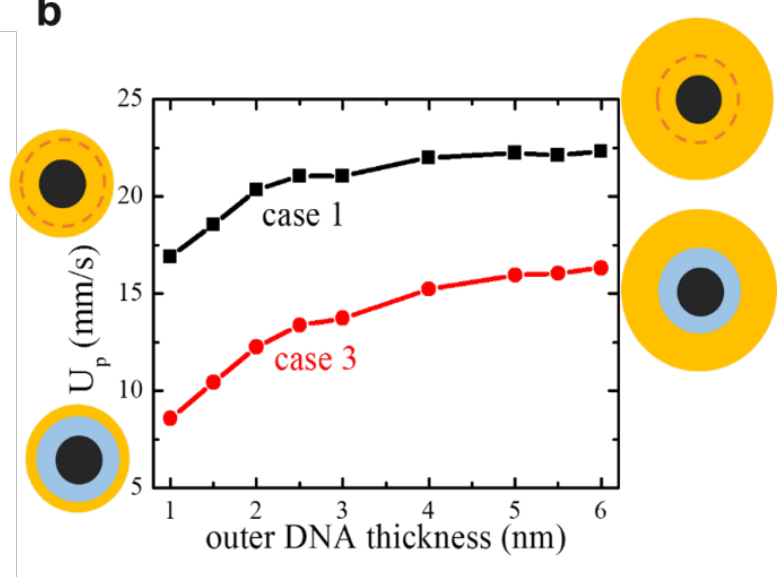

Figure 6. a) Measured particle velocities for the aptamer in the absence (Blue) and presence of metal ions (Red). b) Numerical predictions of translocation velocity of particles containing two layers. The thickness of the outer layer was systematically varied for case 1 and case 3. The thickness of the inner layer is fixed at $3 \mathrm{~nm}$, corresponding to the aptamer binding $\mathrm{Hg}^{2+}$.

\section{Conclusions}

Here we have functionalised particles with metal binding DNA aptamers creating a simple assay for $\mathrm{Hg}^{2+}$ and $\mathrm{Pb}^{2+}$ that is capable of being run in a range of electrolyte concentrations, equivalent to river to sea water conditions. The models show how the charge density around the particles surface effects the relative velocity through nanopore. The changes in particle velocity are relative to the magnitude of the change in charge density of the DNA, which is related to the charge on the metal ion as well as the conformational change upon binding. By developing a dual aptamer which binds to the metal ions in two locations, one next to the particles surface and the second, on the edge of the DNA sequence adjacent to the solution we have shown both experimentally and through modelling how the charge density around the particle can influence the velocity. The modelling revealed that ionic concentrations outside the particle are influenced by the charge density of the outer layer only and the electrokinetic velocity is dependent on the ionic concentrations within the whole thickness of the outer layer. The results here have implications for future DNA-Particles RPS assays, where by understanding the distribution of charge around the material will influence the observed velocities. Experiments presented here were performed with magnetic particles, so that in future the process can be automated, allowing the sample to be extracted and analysed with minimum user interaction. 


\section{Experimental}

\section{Materials and methods}

Poly(ethyleneimine), PEI, (Mw $750000 \mathrm{~g} \mathrm{~mol}^{-1}$, analytical standard, $50 \%$ wt., P3143), poly(acrylic acid-co-maleic acid) (PAAMA, Mw $\sim 3000 \mathrm{~g} \mathrm{~mol}^{-1} 50 \%$ wt., 416053), lead(II) nitrate $\left(\mathrm{Pb}\left(\mathrm{NO}_{3}\right)_{2}, \mathrm{ACS}\right.$ reagent, $\left.\geq 99.0 \%, \mathrm{L7281}\right)$, mercury (II) chloride $\left(\mathrm{HgCl}_{2}\right.$, ACS reagent, $\left.\geq 99.5 \%, \mathrm{M} 6529\right)$, sodium hydroxide $(\mathrm{NaOH}$, reagent grade, $\geq 98 \%$, S5881), (2-(N-Morpholino)ethanesulfonic acid hydrate (MES hydrate, $\geq 99.5 \%, \mathrm{M} 2933$ ) and $\mathrm{N}$-Hydroxysulfosuccinimide sodium salt (NHS, $\geq 98.5 \%, 56485$ ) were purchased from Sigma Aldrich, UK. Tunable conical pores (NP200s) and carboxylated polystyrene particles (210 nm in diameter, denoted CPC200) were purchased from Izon Science, Christchurch, NZ. Potassium chloride $(\mathrm{KCl},>99 \%$, $\mathrm{P} / 4240 / 60)$, sodium chloride ( $\mathrm{NaCl}$, analytical grade, S/3160/60) and Potassium hydroxide $(\mathrm{KOH}, 0.1 \mathrm{M},>85 \%, \mathrm{P} / 5600 / 60)$ were purchased from Fisher Scientific, UK. Hydrochloric acid ( $\mathrm{HCl}, 0.5 \mathrm{M}, 37 \%)$ was purchased from WWR, UK.1-ethyl-3-(3dimethylaminopropyl)carbodiimide hydrochloride (EDC, 22980) was purchased from Thermo Scientific, UK. Reagents were prepared in purified water with a resistance of 18.2 $\mathrm{M} \Omega \mathrm{cm}$ (Maxima). $\mathrm{pH}$ of solutions were altered using $\mathrm{HCl}$ and $\mathrm{KOH}$. The solutions were measured using a Mettler Toledo easy five $\mathrm{pH}$ meter with a Mettler Toledo InLab micro electrode.

\section{Custom DNA oligonucleotides}

The oligonucleotides used in this study were purchased as lyophilised powders from Sigma Aldrich, UK. The sequences were synthesised with an amine functional group at the 3' end: 5'-TTCTTTCTTCGCGTTGTTTGTT-3' (mercury binding aptamer), 5'GGAAGGTGTGGAAGG-3' (lead binding aptamer, TBAA), 5'GGAAATGTGGAAGG-3' (lead control aptamer), 5'TTCTTTCTTCGGGAAGGTGTGGAAGGCGTTGTTTGTT-3' (lead and mercury binding aptamer), 5'-TTCTTTCTTGAGGTTGTTTGTT-3' (loop 4), 5'TTCTTTCTTCGGAGGGGAACGTTGTTTGTT-3' (loop 8) and 5'TTCTTTCTTCGGGAAGTGGAAGCGTTGTTTGTT-3' (loop 11).

\section{DNA functionalised particles}

Superparamagnetic particles were purchased from Ademtech, France (150 nm in diameter, 0211). The particles were modified using PEI and PAAMA to ensure a carboxyl group was present on the particles. Particles were taken from stock $(50 \mu \mathrm{L})$ and suspended in PEI $\left(1 \mathrm{~mL}, 5 \%\right.$ in $\left.\mathrm{H}_{2} \mathrm{O}\right)$. The solution was placed on the rotary wheel for 30 minutes. The solution was vortexed for 5 minutes at $10000 \mathrm{rpm}$, the PEI solution removed from the particles and replaced with water. The sample was vortexed and sonicated until the particles were fully dispersed. This wash step was repeated twice to ensure all excess $\mathrm{PEI}$ had been removed. The PEI coated particles were suspended in PAAMA ( $5 \%$ in $50 \mathrm{mM} \mathrm{NaCl}$ ) for 30 minutes and placed on the rotary wheel. The same process for removing the excess PEI was used. The particles were then stored at $2-4^{\circ} \mathrm{C}$ in water. Carboxyl polystyrene particles (303 
$\mathrm{nm}$ in diameter,11839) were purchased from Bangs Laboratories, US and used without any modification.

Each aptamer was dissolved in 100mM MES buffer ( $\mathrm{pH} 5.7$ ) containing $1 \mathrm{mg} \mathrm{ml}^{-1}$ EDC and $1 \times 10^{10}$ carboxylated particles/ $\mathrm{mL}$. The final concentration of DNA was $410 \mathrm{nM}$. The solution containing the particles was placed on the rotary wheel for 1 hour. To remove an excess DNA, the solutions containing the magnetic $150 \mathrm{~nm}$ particles were placed in a MagRack (Life Sciences). After 5 minutes, the particles had formed a visible cluster and the solution was removed and replaced with an equal volume of $\mathrm{NaCl}(20-500 \mathrm{mM})$. The solutions containing the $300 \mathrm{~nm}$ particles were centrifuged for 10 minutes at $10000 \mathrm{rpm}$. The solution was removed from the pellet of particles and replaced with equal volume of $\mathrm{NaCl}(20-500 \mathrm{mM})$.

\section{Metal ion extraction}

A lead (II) solution ( $1 \mu \mathrm{M})$ was prepared from $\mathrm{Pb}\left(\mathrm{NO}_{3}\right)_{2}$ in a range of ionic strengths $(20-500 \mathrm{mM} \mathrm{NaCl})$. The solution was further diluted in the same ionic strength to give a range of lead concentrations (0.05- $100 \mathrm{nM})$. A $0.1 \mathrm{nM}$ Lead (II) solution was prepared in $\mathrm{KCl}(500 \mathrm{mM})$. A mercury (II) solution $(1000 \mathrm{nM})$ was prepared from $\mathrm{HgCl}_{2}$ in a range of ionic strengths $(20-500 \mathrm{mM} \mathrm{NaCl})$. The solution was further diluted in the same ionic strength to give a range of mercury concentrations (20 $500 \mathrm{nM})$. A copper (II) solution $(1 \mu \mathrm{M})$ was prepared from $\mathrm{CuCl}_{2} \cdot \mathrm{H}_{2} \mathrm{O}$ in $\mathrm{NaCl}(100$ $\mathrm{mM})$. Aptamer modified particles $\left(1 \times 10^{9}\right.$ particles $\left.\mathrm{mL}^{-1}\right)$ were added to the solutions of desired metal ion concentration and ionic strength. The solutions were vortexed for $30 \mathrm{~s}$ and placed on the rotary wheel for 1 hour.

\section{Multiplex analysis of lead and mercury}

$150 \mathrm{~nm}$ particles with $\mathrm{Hg}$ binding aptamer and $300 \mathrm{~nm}$ particles with $\mathrm{Pb}$ binding aptamer were prepared as described above. The functionalised particles were placed into vials at a concentration of $1 \times 10^{9}$ particles $\mathrm{mL}^{-1}$ and lead (20 nM) and mercury $(20 \mathrm{nM})$ were added, the electrolyte concentration was $20 \mathrm{mM}$. The samples were placed on the rotary wheel for 1 hour.

\section{TRPS Set-up}

All measurements were conducted using the qNano (Izon Science, NZ) combing tunable nanopores with data capture and analysis software, Izon Control Suite v.3.1. The lower fluid cell contains the electrolyte $(75 \mu \mathrm{L})$. The particle is suspended in the same electrolyte and placed in the upper fluid cell $(40 \mu \mathrm{L})$. Prior to analysis all samples were vortexed and sonicated for $30 \mathrm{~s}$. After each sample run, the system was washed by placing $40 \mu \mathrm{L}$ of electrolyte into the upper fluid cell several times with various pressures applied to ensure there were no residual particles remaining and therefore no cross contamination between samples. As multiple pores were required throughout the set of experiments we ensured where possible they had comparable pore dimensions. To do this we used the same dimensions of pores as supplied by the manufacturer. Due to the polyurethane material and manufacturing process, some variance is expected in the size. To compensate for this we matched the base 
line current within $5 \%$, as well as running control samples, blanks and calibrations beads, to allow for comparison between data sets. From the baseline current and from data supplied by the manufacturer we calculate the pores to be $780 \mathrm{~nm}$ in diameter at small opening and $58 \mu \mathrm{m}$ in diameter at the bottom, with a thickness of $250 \mu \mathrm{m}$. The zeta potential of the pore wall is $-11 \mathrm{mV}^{55}$

\section{Particle translocation velocity}

The relative velocity of the particle can then be calculated from the pulse width, Figure S3. Multiple time points are recorded along the peak and are donated $T_{0.90}$, $\mathrm{T}_{0.80}, \mathrm{~T}_{0.70}$ etc., and the reciprocal of the average time from each point can be used to calculate the relative particle velocity.

The method uses the resistive pulse to calculate the relative velocity. For each blockade, the time at which the peak occurs is defined as $\mathrm{T}_{1.0}$ (time at $100 \%$ of peak magnitude) and the maximum magnitude of the pulse (relative to the local baseline resistance) is recorded as $d R_{\max }$. In the example shown in Figure S3 sections, $60 \%$, $50 \%, 40 \%$, and $30 \%$ of $\mathrm{d} R_{\max }$ are displayed. The duration from $\mathrm{T}_{1.0}$ to each of these sections is defined at $T_{0.60}, T_{0.50}, T_{0.40}$ and $T_{0.30}$. When the proportional blockade magnitude is equal for any given particles (small or large), these particles are at the same position within the pore. Hence, the relative magnitude is an indicator of the particle position within the pore.

\section{Models and Simulations}

The electrokinetic translocation of a DNA or aptamer modified nanoparticle through a conical nanopore is modelled using the coupled Poisson-Nernst-Planck and

Stokes-Brinkman equations. ${ }^{53,56,57}$ On the basis of the assumption of quasi-steady state, the translocation velocity of DNA or aptamer nanoparticle can be determined by a balance of the electrical and hydrodynamic forces. ${ }^{58}$ These highly coupled mathematical model is numerically solved by finite element package COMSOL Multiphysics. Details of governing equations, boundary conditions, and numerical implementation are provided in the Supporting Information.

\section{Acknowledgements}

ZS and CYL acknowledge Horizon Development, LLC for financial support.

\section{Conflict of Interest}

The authors declare no conflict of interest

\section{Supporting Information Available}

Supporting Information Available: The following files are available free of charge. Examples of raw current traces with pulse magnitude distributions for particles, DNA functionalised particles, average particle velocities for DNA functionalised particles in different electrolyte solutions, calculations for determining the relative velocities and charge densities around each particles and Schematic illustration of a system 
modelled where a dual modified nanoparticle is placed at the tip opening of the conical pore. This material is available free of charge via the internet at http://pubs.acs.org

\section{References}

(1) Ellington, A. D.; Szostak, J. W. In Vitro Selection of RNA Molecules That Bind Specific Ligands. Nature 1990, 346, 818-822.

(2) Tuerk, C.; Gold, L. Systematic Evolution of Ligands by Exponential Enrichment : RNA Ligands to Bacteriophage T4 DNA Polymerase. Science. 1990, 249, 505-510.

(3) Knight, C. G.; Platt, M.; Rowe, W.; Wedge, D. C.; Khan, F.; Day, P. J. R.; McShea, A.; Knowles, J.; Kell, D. B. Array-Based Evolution of DNA Aptamers Allows Modelling of an Explicit Sequence-Fitness Landscape. Nucleic Acids Res. 2009, 37, e6.

(4) Platt, M.; Rowe, W.; Wedge, D. C.; Kell, D. B.; Knowles, J.; Day, P. J. R. Aptamer Evolution for Array-Based Diagnostics. Anal. Biochem. 2009, 390, 203-205.

(5) Cox, J. C.; Rudolph, P.; Ellington, A. D. Automated RNA Selection. Biotechnol Prog 1998, 14, 845-850.

(6) Bunka, D. H. J.; Stockley, P. G. Aptamers Come of Age - at Last. Nat Rev Micro 2006, 4 , 588-596.

(7) Keefe, A. D.; Pai, S.; Ellington, A. Aptamers as Therapeutics. Nat Rev Drug Discov 2010, 9, 537-550.

(8) Cho, E. J.; Lee, J.-W.; Ellington, A. D. Applications of Aptamers as Sensors. Annu. Rev. Anal. Chem. (Palo Alto. Calif). 2009, 2, 241-264.

(9) Levy-Nissenbaum, E.; Radovic-Moreno, A. F.; Wang, A. Z.; Langer, R.; Farokhzad, O. C. Nanotechnology and Aptamers: Applications in Drug Delivery. Trends Biotechnol. 2008, 26, 442-449.

(10) Mairal, T.; Cengiz Özalp, V.; Lozano Sánchez, P.; Mir, M.; Katakis, I.; O'Sullivan, C. K. Aptamers: Molecular Tools for Analytical Applications. Anal. Bioanal. Chem. 2008, 390, 989-1007.

(11) Willner, I.; Shlyahovsky, B.; Zayats, M.; Willner, B. DNAzymes for Sensing\{,\} Nanobiotechnology and Logic Gate Applications. Chem. Soc. Rev. 2008, 37, 1153-1165.

(12) Shim, J.; Humphreys, G. I.; Venkatesan, B. M.; Munz, J. M.; Zou, X.; Sathe, C.; Schulten, K.; Kosari, F.; Nardulli, A. M.; Vasmatzis, G.; Bashir, R. Detection and Quantification of Methylation in DNA Using Solid-State Nanopores. Sci. Rep. 2013, 3, 1389.

(13) Branton, D.; Deamer, D. W.; Marziali, A.; Bayley, H.; Benner, S. A.; Butler, T.; Di Ventra, M.; Garaj, S.; Hibbs, A.; Huang, X.; Jovanovich, S. B.;Krstic, P. S.; Lindsay, S.; Ling, X. S.; Mastrangelo, C. H.; Meller, A.; Oliver, J.S.; Pershin, Y. V.; Ramsey, J. M.; Riehn, R.; et al. The Potential and Challenges of Nanopore Sequencing. Nat Biotech 2008, 26, 1146-1153.

(14) Rotem, D.; Jayasinghe, L.; Salichou, M.; Bayley, H. Protein Detection by Nanopores Equipped with Aptamers. J. Am. Chem. Soc. 2012, 134, 27812787.

(15) Ali, M.; Nasir, S.; Ensinger, W. Bioconjugation-Induced Ionic Current Rectification in Aptamer-Modified Single Cylindrical Nanopores. Chem. 
Commun. 2015, 51, 3454-3457.

(16) Platt, M.; Willmott, G. R.; Lee, G. U. Resistive Pulse Sensing of AnalyteInduced Multicomponent Rod Aggregation Using Tunable Pores. Small 2012, 8, 2436-2444.

(17) Siwy, Z.; Apel, P.; Dobrev, D.; Neumann, R.; Spohr, R.; Trautmann, C.; Voss, K. Ion Transport through Asymmetric Nanopores Prepared by Ion Track Etching. Nucl. Instruments Methods Phys. Res. Sect. B Beam Interact. with Mater. Atoms 2003, 208, 143-148.

(18) Siwy, Z.; Trofin, L.; Kohli, P.; Baker, L. A.; Trautmann, C.; Martin, C. R. Protein Biosensors Based on Biofunctionalized Conical Gold Nanotubes. J. Am. Chem. Soc. 2005, 127, 5000-5001.

(19) Kozak, D.; Anderson, W.; Vogel, R.; Chen, S. Simultaneous Size and $\zeta-$ Potential Measurements of Individual Nanoparticles in Dispersion Using SizeTunable Pore Sensors. ACS nano 2012, 6, 6990-6997.

(20) Blundell, E. L. C. J.; Vogel, R.; Platt, M. Particle-by-Particle Charge Analysis of DNA-Modi Fi Ed Nanoparticles Using Tunable Resistive Pulse Sensing. 2015.

(21) Mayne, L. J.; Christie, S. D. R.; Platt, M. A Tunable Nanopore Sensor for the Detection of Metal lons Using Translocation Velocity and Biphasic Pulses. Nanoscale 2016, 8, 19139-19147.

(22) Billinge, E. R.; Platt, M. Aptamer Based Dispersion Assay Using Tunable Resistive Pulse Sensing (TRPS). Anal. Methods 2015, 7, 8534-8538.

(23) Billinge, E. R.; Platt, M. Multiplexed, Label-Free Detection of Biomarkers Using Aptamers and Tunable Resistive Pulse Sensing (AptaTRPS). Biosens. Bioelectron. 2015, 68, 741-748.

(24) Billinge, E. R.; Broom, M.; Platt, M. Monitoring Aptamer-Protein Interactions Using Tunable Resistive Pulse Sensing. Anal. Chem. 2013, 86, 1030-1037.

(25) Alsager, O. a; Kumar, S.; Willmott, G. R.; McNatty, K. P.; Hodgkiss, J. M. Small Molecule Detection in Solution via the Size Contraction Response of Aptamer Functionalized Nanoparticles. Biosens. Bioelectron. 2014, 57, 262268.

(26) Billinge, E. R.; Platt, M. Multiplexed, Label-Free Detection of Biomarkers Using Aptamers and Tunable Resistive Pulse Sensing (AptaTRPS). Biosens. Bioelectron. 2015, 68.

(27) Blundell, E. L. C. J.; Mayne, L. J.; Christie, S. D. R.; Platt, M. Protein Detection Using Tunable Pores: Resistive Pulses and Current Rectification. Faraday Discuss. 2016, No. 193, 487-505.

(28) Billinge, E. R.; Broom, M.; Platt, M. Monitoring Aptamer-Protein Interactions Using Tunable Resistive Pulse Sensing. Anal. Chem. 2014, 86.

(29) Menestrina, J.; Yang, C.; Schiel, M.; Vlassiouk, I.; Siwy, Z. S. Charged Particles Modulate Local Ionic Concentrations and Cause Formation of Positive Peaks in Resistive-Pulse-Based Detection. J. Phys. Chem. C 2014, 118, 2391-2398.

(30) Weatherall, E.; Willmott, G. R. Conductive and Biphasic Pulses in Tunable Resistive Pulse Sensing. J. Phys. Chem. B 2015, 119, 5328-5335.

(31) Lan, W.-J.; Kubeil, C.; Xiong, J.-W.; Bund, A.; White, H. S. Effect of Surface Charge on the Resistive Pulse Waveshape during Particle Translocation through Glass Nanopores. J. Phys. Chem. C 2014, 118, 2726-2734.

(32) Qiu, Y.; Yang, C.; Hinkle, P.; Vlassiouk, I. V; Siwy, Z. S. Anomalous Mobility of Highly Charged Particles in Pores. Anal. Chem. 2015, 87, 8517-8523.

(33) Siwy, Z.; Heins, E.; Harrell, C. C.; Kohli, P.; Martin, C. R. Conical-Nanotube 
Ion-Current Rectifiers: The Role of Surface Charge. J. Am. Chem. Soc. 2004, 126, 10850-10851.

(34) Buchsbaum, S. F.; Nguyen, G.; Howorka, S.; Siwy, Z. S. DNA-Modified Polymer Pores Allow pH- and Voltage-Gated Control of Channel Flux. J. Am. Chem. Soc. 2014, 136, 9902-9905.

(35) Xiang, Y.; Lu, Y. DNA as Sensors and Imaging Agents for Metal Ions. Inorg. Chem. 2014, 53, 1925-1942.

(36) Zhou, W.; Saran, R.; Liu, J. Metal Sensing by DNA. Chem. Rev. 2017, 117, 8272-8325.

(37) Yang, D.; Liu, X.; Zhou, Y.; Luo, L.; Zhang, J.; Huang, A.; Mao, Q.; Chen, X.; Tang, L. Aptamer-Based Biosensors for Detection of Lead Ion: A Review. Anal. Methods 2017, 9, 1976-1990.

(38) Sen, D.; Gilbert, W. A Sodium-Potassium Switch in the Formation of FourStranded G4-DNA. Nature. 1990, pp 410-414.

(39) Jing, N.; Rando, R. F.; Pommier, Y.; Hogan, M. E. Ion Selective Folding of Loop Domains in a Potent Anti-HIV Oligonucleotide. Biochemistry 1997, 36, 12498-12505.

(40) Kim, B. G.; Long, J.; Dubins, D. N.; Chalikian, T. V. Ionic Effects on VEGF GQuadruplex Stability. J. Phys. Chem. B 2016, 120, 4963-4971.

(41) Kiziria, E.; Gorgoshidze, M.; Gogichaishvili, S.; Sokhadze, V.; Khachidzr, D.; Kiladze, M.; Lomidze, E.; Barbakadze, S.; Tvauri, G.; Monaselidze, J. Influence of K + Ions on Thermodynamic Stability of DNA G-Quadruplex; 2017; Vol. 11.

(42) Smirnov, I. V.; Kotch, F. W.; Pickering, I. J.; Davis, J. T.; Shafer, R. H. Pb EXAFS Studies on DNA Quadruplexes: Identification of Metal Ion Binding Site. Biochemistry 2002, 41, 12133-12139.

(43) Torigoe, H.; Ono, A.; Kozasa, T. Hgll Ion Specifically Binds with T:T Mismatched Base Pair in Duplex DNA. Chem. - A Eur. J. 2010, 16, 1321813225.

(44) Blundell, E. L. C. J.; Vogel, R.; Platt, M. Particle-by-Particle Charge Analysis of DNA-Modified Nanoparticles Using Tunable Resistive Pulse Sensing. Langmuir 2016, 32, 1082-1090.

(45) van Dorp, S.; Keyser, U. F.; Dekker, N. H.; Dekker, C.; Lemay, S. G. Origin of the Electrophoretic Force on DNA in Solid-State Nanopores. Nat. Phys. 2009, $5,347$.

(46) Keyser, U. F.; van Dorp, S.; Lemay, S. G. Tether Forces in DNA Electrophoresis. Chem. Soc. Rev. 2010, 39, 939-947.

(47) He, Y.; Tsutsui, M.; Fan, C.; Taniguchi, M.; Kawai, T. Controlling DNA Translocation through Gate Modulation of Nanopore Wall Surface Charges. ACS Nano 2011, 5, 5509-5518.

(48) Zhang, M.; Yeh, L.-H.; Qian, S.; Hsu, J.-P.; Joo, S. W. DNA Electrokinetic Translocation through a Nanopore: Local Permittivity Environment Effect. J. Phys. Chem. C 2012, 116, 4793-4801.

(49) Kozak, D.; Anderson, W.; Vogel, R.; Trau, M. Advances in Resistive Pulse Sensors: Devices Bridging the Void between Molecular and Microscopic Detection. Nano Today 6, 531-545.

(50) Song, Y.; Murray, R. W. Dynamics and Extent of Ligand Exchange Depend on Electronic Charge of Metal Nanoparticles. J. Am. Chem. Soc. 2002, 124, 7096-7102.

(51) Platt, M.; Rowe, W.; Knowles, J.; Day, P. J.; Kell, D. B. Analysis of Aptamer 
Sequence Activity Relationships. Integr. Biol. 2009, 1, 116-122.

(52) Stevens, P. W.; Henry, M. R.; Kelso, D. M. DNA Hybridization on Microparticles: Determining Capture-Probe Density and Equilibrium Dissociation Constants. Nucleic Acids Res. 1999, 27, 1719-1727.

(53) Ohshima, H. Electrophoresis of Soft Particles. Adv. Colloid Interface Sci. 1995, 62, 189-235.

(54) Yeh, L.-H.; Zhang, M.; Qian, S.; Hsu, J.-P. Regulating DNA Translocation through Functionalized Soft Nanopores. Nanoscale 2012, 4, 2685-2693.

(55) Blundell, E. L. C. J.; Vogel, R.; Platt, M. Particle-by-Particle Charge Analysis of DNA-Modified Nanoparticles Using Tunable Resistive Pulse Sensing. Langmuir 2016, 32. 1082-1090

(56) Duval, J. F. L.; Gaboriaud, F. Progress in Electrohydrodynamics of Soft Microbial Particle Interphases. Curr. Opin. Colloid Interface Sci. 2010, 15, 184-195.

(57) Lin, C.-Y.; Hsu, J.-P.; Yeh, L.-H. Rectification of Ionic Current in Nanopores Functionalized with Bipolar Polyelectrolyte Brushes. Sensors Actuators $B$ Chem. 2018, 258, 1223-1229.

(58) Qiu, Y.; Lin, C.-Y.; Hinkle, P.; Plett, T. S.; Yang, C.; Varghese Chacko, J.; Digman, M. a; Yeh, L.-H.; Hsu, J.-P.; Siwy, Z. S. Highly Charged Particles Cause a Larger Current Blockage in Micropores Compared to Neutral Particles. ACS Nano 2016, 10, 8413-8422.

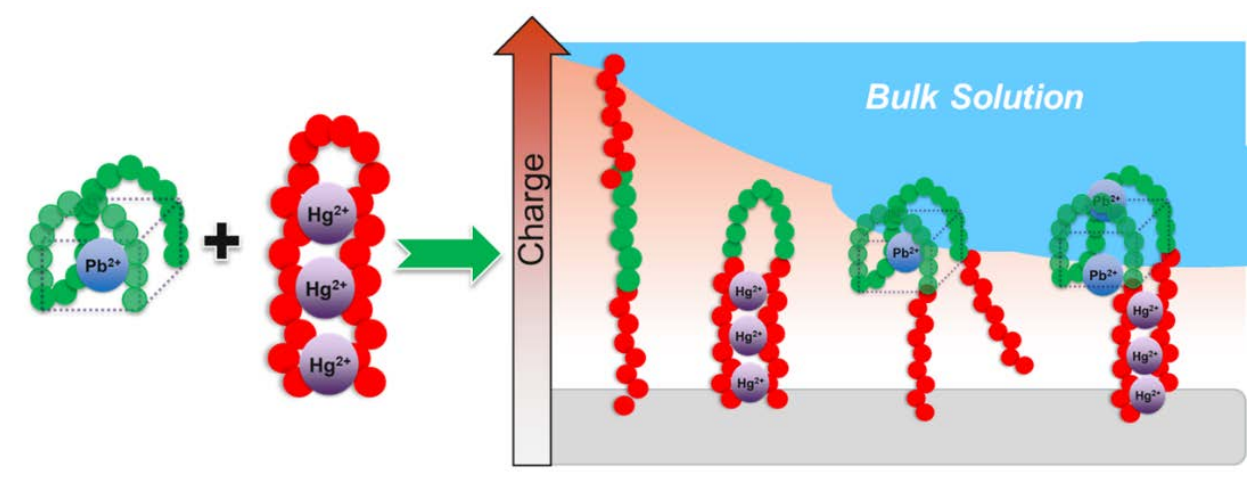

